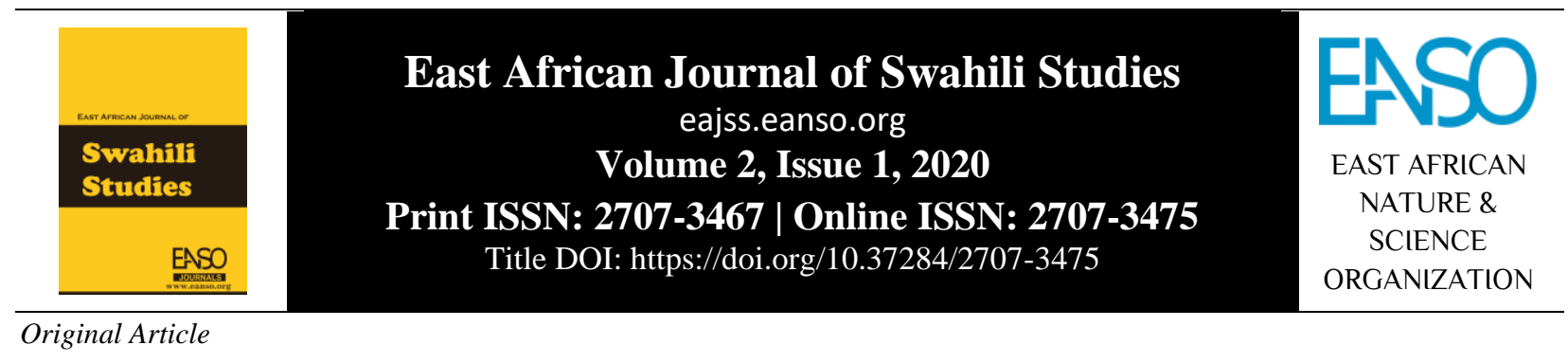

\title{
Mifumo ya Kijamii katika Tamthilia Teule za Kiswahili
}

\author{
Samson Ezekiel Oketch ${ }^{*}$, Dkt. Beverlyne Asiko Ambuyo ${ }^{2}$ \& Dkt. Rosemary Makokha ${ }^{1}$ \\ ${ }^{1}$ Chuo Kikuu cha Mount Kenya, S. L. P. $441-40200$, Kisii, Kenya. \\ ${ }^{2}$ Chuo Kikuu cha Maseno, S. L. P. 333 - 40105, Maseno, Kenya. \\ *Author for Correspondence email: samson.oketchezekiel@gmail.com
}

Article DOI: https://doi.org/10.37284/eajss.2.1.135

\section{Article history:}

Received: 23 Oct 2019

Accepted: 01 Nov 2019

Published: 10 Apr 2020

Keywords:

Mifumo,

Jamii,

Janajike,

Mifumo Jamii,

Umbuji.

\section{IKISIRI}

Mifumo ya kijamii inajitokeza waziwazi katika fasihi andishi ya Kiswahili. Mifumo hiyo huwakilisha matukio ya fasihi andishi na jinsi yanavyoichora na kwa mapana kuwa kioo cha jamii. Tafiti nyingi zilizofanywa zinahusu vipengele vya fasihi kama vile maudhui, matumizi ya lugha, wahusika na mandhari katika fasihi. Katika tafiti hizo, watafiti hawajaonyesha mifumo jamii inayojitokeza kutokana na vipengele hivi. Utafiti huu umezama na kiutathimini mifumo hii kupitia kwa tamthilia teule za Kiswahili ambazo ni: tamthilia ya Pango (2003) kilichoandikwa na Wamitila, Kifo Kisimani (2008) kilichoandikwa na Kithaka Wa Mberia na Mstahiki Meya (2009) kilichoandikwa na Timothy Arege. Mifumo iliyotafitiwa katika utafiti huu ni: mfumo wa utawala na siasa, wa uchumi katika jamii, wa utamaduni, wa imani na dini, ule wa tabaka za jamii na ule wa elimu ya jamii. Utafiti huu umeongozwa na madhumuni mahsusi yafutayo: kujadili maudhui yanayodhihirisha mifumo ya jamii katika tamthilia teule; kuchanganua mitindo ya lugha inavyodhihirisha mifumo ya kijamii kutoka tamthilia teule; na kuchunguza wahusika katika uhusika wao kwenye mandhari maalumu wanavyojenga ploti inayochangia kuweka wazi mifumo ya kijamii kutoka tamthilia teule. Kiunzi cha nadharia ya uchunguzi kimejengwa na nadharia za uhalisia na nadharia ya umtindo. Mihimili ya nadharia ya uhalisia ikiwemo masuala ya utawala na uongozi, yale ya vijana, udhanifu, ukosefu wa ajira na masuala ya jinsia imebainishwa kadri ilivyojitokeza kupitia tamthilia teule. Mbali na nadharia za uhalisia na umtindo nadharia ya ukoloni mamboleo imetumika kuelezea hali katika tamthilia hasa utambuzi wa mifumo jamii inayotokana na matokeo ya baada ya uhuru. Utafiti umeongozwa na tamthilia 26 zilizoandikwa nchini Kenya na Tanzania tangu mwaka wa 2000. Tamthilia 26 zilizotumika ni zile zilizoandikwa na wazawa asilia wa Afrika Mashariki 
na kati kufikia mwaka wa 2014. Asilimia kumi ya idadi hiyo ilipeana sampuli ya mbili nukta sita (2.6) ambacho ni kifani tosha cha tamthilia tatu. Utafiti huu umejikita Kenya na Tanzania kwa kigezo kuwa mbali na Kiswahili kuchimbukia na kuenea zaidi katika janibu hizi, imebainika pia kuwa sera na sheria za kikatiba za nchi hizi zimekipa Kiswahili kipao mbele. Orodha ya uchuzaji imetumika kukusanya data. Uchanganuzi wa data ulitumia yaliyomo. Utafiti huu unatarajiwa kuibua mawazo mapya kuhusu mifumo ya kijamii inayotokana na fasihi na hivyo kuchangia katika ukuaji wa elimu.

\section{APA CITATION}

Oketch, S., Ambuyo, B., \& Makokha, R. (2020). Mifumo ya Kijamii katika Tamthilia Teule za Kiswahili. East African Journal of Swahili Studies, 2(1), 8-18. https://doi.org/10.37284/eajss.2.1.135.

\section{CHICAGO CITATION}

Oketch, Samson, Beverlyne Ambuyo, and Rosemary Makokha. 2020. "Mifumo Ya Kijamii Katika Tamthilia Teule Za Kiswahili”. East African Journal of Swahili Studies 2 (1), 8-18. https://doi.org/10.37284/eajss.2.1.135.

\section{HARVARD CITATION}

Oketch, S., Ambuyo, B. and Makokha, R. (2020) "Mifumo ya Kijamii katika Tamthilia Teule za Kiswahili", East African Journal of Swahili Studies, 2(1), pp. 8-18. doi: 10.37284/eajss.2.1.135.

\section{IEEE CITATION}

S. Oketch, B. Ambuyo, and R. Makokha, "Mifumo ya Kijamii katika Tamthilia Teule za Kiswahili”, EAJSS, vol. 2, no. 1, pp. 8-18, Apr. 2020.

\section{MLA CITATION}

Oketch, Samson, Beverlyne Ambuyo, and Rosemary Makokha. "Mifumo Ya Kijamii Katika Tamthilia Teule Za Kiswahili". East African Journal of Swahili Studies, Vol. 2, no. 1, Apr. 2020, pp. 8-18, doi:10.37284/eajss.2.1.135.

\section{UTANGULIZI}

Fasihi ina majukumu mbalimbali ambayo yana umuhimu kwa jamii. Njogu na Chimerah (2006) wanasema kuwa mojawapo ya majukumu hayo ni kuichora jamii jinsi ilivyo. Ili ipate ufanisi katika jukumu hili, fasihi sharti ihusishe wanajamii katika mazingira yao ya kawaida, shughuli wazifanyazo ili kujimudu kila siku, utawala, uongozi na siasa katika jamii, utamaduni, itikadi, dini na falsafa zao za maisha. Mambo hayo yote huhusisha mipangilio maalumu. Wamitila (2004) anaiita mipangilio hii kuwa ya kimawazo na kimaelezo itokanayo na dhana na kanuni zilizo na lengo la kusaidia katika kazi ya uhakiki, uchambuzi na uchanganuzi wa kazi ya fasihi. Mipango hiyo huwasaidia binadamu kuzikabili changamoto wanazokumbana nazo maishani. Mifumo ya kijamii ambayo imechunguzwa katika utafiti huu imebainisha waziwazi kupitia kwa moja kati ya tanzu nne za fasihi andishi kuwa ni kweli kwamba yaliyoandikwa kwenye fasihi pia hutendeka katika jamii.
Kutokana na matokeo ya utafiti hoja kuwa fasihi ni kioo cha jamii inapata mashiko. Haya ni kwa sababu uchunguzi huu umefanyika katika misingi thabiti ambayo Wamitila (2010) anasema kuwa sharti ijengeke katika mifumo na nadharia thabiti ambazo hutoa mwongozo katika matumizi ya kuichunguza kazi ya fasihi. Uchunguzi wa mifumo ya jamii umefanyika kwa misingi ya nadharia ya uhalisia. Baadhi ya nguzo za nadharia hii ni kuwa inahusu maisha ya binadamu katika ukweli (Ntarangwi, 2004). Ni kutokana na mantiki hiyo ambapo utafiti huu umechunguza mifumo hiyo katika uhalisia na dhana zake. Katika uchunguzi huu, baadhi ya vitabu teule vya tamthilia vilivyowezesha utambuzi wa nafasi ya fasihi andishi katika jamii vimetumika. Hili limepelekea kuelewa kuwa si yote yaliyoandikwa katika fasihi ambayo ni bunilizi bali mengine huwa ya ukweli na hasa yanapohusisha maisha halisi ya binadamu. Mwanafasihi hutumika kama chombo cha kupitisha ujumbe kwa njia fiche kwa wanajamii, labda kuonya, kuburudisha ama kuelimisha jamii. Mbali na hayo, uchunguzi umebainisha jinsi mifumo ya kijamii inaweza kuleta mshikamano na manufaa 
mengine mengi kwa wanajamii na kuepusha migogoro na mivutano ambayo ingeweza kuepukika kwa njia za amani kwa mfano; mizozo ya kijamii iwe ya kiuchumi, kiutawala ama kitamaduni yaweza kusuluhishwa bila kuwepo migongano yoyote baina ya wanajamii.

Wanajamii na viongozi wa kiimla wanaweza kujifunza kuwa uchu wa madaraka na kujilimbikizia mali ya umma mara nyingi havileti amani wala maendeleo na aghalabu viongozi hawa hawatimizi malengo yao bila upinzani, manung'uniko na wakati mwingine kupenduliwa. Wanajamii pia wanaweza kujifunza kuwa kuna tamaduni zilizopitwa na wakati ambazo hazileti tija yoyote zikizingatiwa. Mfano mwafaka ni kutowachagua wanawake katika ngazi za uongozi huku ikiwa ni kwa misingi ya jinsia. Ukweli ni kuwa kuna usawa na mwanamke kama binadamu yeyote huenda akafanya vizuri zaidi kuliko hao wanaume wanaowachagua. Utafiti huu umebainisha haya yote huku tukitumia vipengele vya lugha kama vile maudhui, fani za lugha zilizotumiwa na wahusika kutoka tamthilia teule katika mandhari yanayotofautiana huku vipengele hivyo vikichanganuliwa kwa nadhara ya umtindo. Utafiti huu umezingatia kipindi cha milenia, yaani kati ya mwaka 2000 na 2014 kwa sababu hiki ndicho kipindi ambacho Muungano wa Umoja wa Mataifa uliweka malengo ya milenia; yakiwemo usawa wa jinsia katika taasisi za elimu, ajira na kupunguza vifo vya watoto wachanga. Hiki ndicho kipindi ambapo pia utandawazi ulikumbatiwa hasa na vijana na mabadiliko mengi yalifanywa katika taasisi na mamlaka za kiserikali. Wananchi nao walipata nguvu na mamlaka ya kisheria na kikatiba kujichagulia viongozi wao kwa njia za kidemokrasia. Nguvu zenyewe zilitokana na harakati za uundaji wa katiba mpya zilizofaulu. Haya ni kwa mujibu wa Katiba ya Kenya sura ya kwanza, sehemu ya kwanza ibara ya pili na tatu (Constitution of Kenya, 2010). Utafiti huu umetumia tamthilia za Pango (2003) cha Wamitila, Kifo Kisimani (2008) cha wa Mberia, na Mstahiki Meya (2009) cha Arege.

Msokile (2003) anasema kuwa mifumo hujengeka kutokana na nadharia za wakati husika. Anatoa mifano ya mifumo ya kale iliyojengeka kutokana na nadharia za wakati huo. Baadhi ya mifano hiyo ni nadharia za karne ya kumi na tano hadi karne ya kumi na saba. Nadharia hizo ni umapokezi na umuundo wa kifasihi. Aidha, kulikuwa na uasilia uliohusisha matendo na matukio ya kifasihi na nguvu za kimazingira na kimaumbile iliyokuwa na mashiko hadi karne ya kumi na tisa. Katika karne hii pia kulizuka nadharia ya urasmi iliyosisitiza malenga kutumia sheria, kanuni na kaida za Kiaristotle katika utunzi na ukariri wa mashairi. Ulimbwende ulifuata kwa karibu nao ukakaidi na kudengua masharti ya kirasmi na kusisitiza uhuru wa mtunzi, ubunifu na jazba katika sanaa. Kutokana na mawazo ya Msokile, nadharia iliyoasisiwa karne ya 19 ya uhalisia iliteuliwa kuwa moja kati ya nadharia tete katika uchunguzi huu kwa sababu inasisitiza ukweli wa maisha katika jamii. Kiunzi cha nadharia kimeundwa na nadharia ya umitindo kwa sababu ni kutokana na mtindo ambapo mifumo jamii huzuka.

Wazo la kuzingatiwa ni kuwa mifumo ya kijamii lazima iwe na athari katika maisha ya wanajamii. Hivyo basi uchunguzi wowote uliohusu maisha ya binadamu yanafaa yawe katika misingi ya ukweli na kuaminika (Ntarangwi, 2004). Kadhalika, uchunguzi ulitumia nadharia za umtindo na ukoloni mamboleo ili kuyakabili mambo ibuka ambayo kwa njia moja au nyingine yana uwezo wa kuiathiri mifumo iliyochunguzwa na maisha ya wanajamii kwa ujumla. Watt (1957) alichangia kuhusu nadharia ya uhalisia aliposema kuwa ilishika kasi karne ya kumi na tisa na ilisisitiza mambo na matukio ya kifasihi kuelezwa kwa ukweli. Chanzo cha ukweli wenyewe ni jamii. Wamitila (2010) anauona ukweli aliouona Watt na kuongezea kuwa ukweli huo waweza kujitokeza kimifumo katika miktadha, mitizamo, itikadi, mielekeo na falsafa wanazozifuata wanajamii na huwa tofauti kutoka jamii moja hadi nyingine na wakati mwingine huweza kutofautiana katika watu binafsi. Hii ndiyo mifumo ya jamii katika ukweli wake na inapodhihirika huwa chombo cha jamii kinachodhihirisha dhima ya fasihi ya kuwa kioo cha jamii, kuleta burudani na kuelimisha wanajamii (Njogu na Chimerah, 2006).

Wamitila (2004) aliorodhesha mifumo ya kijamii kuwa: siasa na utawala, uchumi wa jamii, utamaduni, itikadi na historia ya jamii husika. Maudhui katika kazi za kifasihi hujengeka kutokana na mifumo hiyo. Maudhui hio hufanikishwa na shughuli wazifanyazo wanajamii, 
lugha wanayoitumia kwenye shughuli hizo na hata wahusika kwenye shughuli hizo. Katika fasihi, mifumo inayojadiliwa hutokana miongoni mwa wanajamii wenyewe. Njogu na Chimerah (2006) wanasema kuwa wahakiki na waandishi hutwikwa jukumu la kuonyesha matukio yatokanayo na wahusika wa kifasihi katika hali halisi na kufichua migogoro iliyomo kwenye jamii ili kupevusha wanajamii kuchukua mitazamo na misimamo katika mambo yanayoendelea ambayo huenda kwa njia moja ama nyingine yakaathiri maisha yao. Katika hali hii, mifumo ya jamii huwa imeathiri mtu binafsi na husababisha fasihi kuwa chombo cha jamii kinachoweka matabaka ya wanajamii paruwanja. Hapa fasihi yenyewe hutumika kwa njia chanya ya kumtia mwanajamii mshawasha wa kuikosoa jamii ambapo mambo hayaendi vizuri, kukemea uovu na uozo unaovumbiwa macho na baadhi ya wanajamii, aghalabu kudadisi na kuikataa mifumo dhalilishi iliyomo kwenye jamii. Mfano wa mifumo dhalilishi ni ile inayobagua watu kwa umri wao, jinsia zao na hata kabila wanamotoka. Katika tasnifu hii, utafiti uliyaangazia haya huku lengo kuu likiwa kuonyesha vile yanachangia katika ukuaji wa fasihi na jamii zetu kwa ujumla. Ni kutokana na tafiti kama hizi ambapo pengo lililopo baina ya matabaka ya jamii linaweza kuwekwa wazi ili mamlaka ziweze kutafuta mbinu mwafaka za kupunguza pengo hilo. Katika tamthilia teule, waandishi walisheheni na kubainisha ukweli katika uhalisia huku wakitumia wahusika kutoa mielekeo halisi ya maisha ya binadamu wa kawaida katika kipindi kinachozungumziwa. Haya yamebainika kutokana na uchanganuzi wa msuko wa matukio kwenye tamthilia teule.

Kwa mujibu wa Gill (2006) msuko ama ploti hutokana na mfuatano wa matukio ya uhusika ili kuleta dhamira na maudhui yaliyokusudiwa na mwandishi wa kazi ya kifasihi. Watt (1957) akirejelea wazo kuwa maisha ya binadamu sharti yaonyeshe ukweli, anasisitiza kuwa ukweli huo uwe wa kuaminika na si wa kibunilizi. Hivyo ni kusema kuwa mifumo ya kifasihi yafaa itokane na matukio ya hakika kutoka kwa jamii husika katika hali halisi. Ntarangwi (2004:34) anaongeza kwamba mbali na uhalisia wa kimfumo kuwakilisha mambo katika fasihi, sharti mifumo hiyo pia iwe ya kweli na endapo ni bunilizi basi iweze kuwa inaaminika.
Nadharia zingine kwenye uchunguzi huu ni umtindo na ukoloni mamboleo. Umtindo ni nadharia ya karne ya ishirini na moja inayohusisha uchanganuzi wa maneno katika usemi. Ukoloni mamboleo kwa mtazamo wa Kwame Nkrumah, katika 'Neo-colonialism, The last stage of Imperialism' anauona kama hatua za mwisho za ubepari (Nkrumah, 1965). Anazidi kusema kuwa ukoloni mamboleo ulianza punde tu baada ya nchi za Kiafrika kujinyakulia uhuru wao miaka ya 1960 hadi 1970. Lengo hasa lilikuwa kuwafanya wachache wanaotawala wamiliki vyanzo vya uchumi. Kutokana na kauli hii, utafiti umebaini kuwa viongozi wetu, yaani wa nchi ambazo hazijapata maendeleo makubwa ya kiviwanda, hujilimbikizia mali ya umma mbali na kuwanyanyasa wananchi wanaowatawala my). Kwa mujibu wa Obeng-Odoom (2013) viongozi hawa hudai nchi zao kuwa huru lakini cha kushangaza ni kwamba wao hutumia zile sera za kikoloni zilizoachwa na wakoloni na pia kuwategemea wakoloni walioondoka katika shughuli zote za kiulinzi na kiuchumi. Wakoloni nao kwa upande mwingine hufaidika kwa kuweka sera zinazowafaidi wananchi wao na chumi zao na aidha wao huweka sera na vikwazo ili wawe na uwezo wa kuzitumia raslimali za nchi walizozitawala pale awali, wakidai kufanya juhudi za kuinua maisha ya wananchi wa nchi husika. Matokeo ya hayo ni vibaraka hao; viongozi wa nchi changa kufaidika pakubwa. Umaskini uliokithiri hutawala mwananchi wa kawaida, ukosefu wa soko za bidhaa wanazozichuma na ukosefu wa ajira. Haya yote yalibainika kutoka kwa msuko wa matukio katika tamthilia teule.

Kupitia mifano na hali katika tamthilia, uchunguzi huu ulibainisha matabaka yalivyojengeka kutokana na ukoloni mamboleo na kupendekeza mwelekeo kwa changamoto zake kwa kutumia wahusika wanaoongoza wanajamii kudai uwajibikaji, utawala bora na kushirikishwa katika maamuzi ya jamii. Ukoloni mamboleo wakati mwingine huchukua sifa za usasa na kuupinga ukale na matokeo yake ni kuzuka kwa utabaka katika jamii. Matabaka yanapotokea katika jamii nadharia ya usasa hutawala jamii ambapo baadhi ya sifa zake ni vijiji kugeuka kuwa miji mikubwa, kukataa tamaduni zilizopitwa na wakati na pia kukataa utamaduni na utawala usioleta mabadiliko hasa maendeleo ya kiviwanda. Wanafilosofia huiona 
nadharia ya usasa kama inayowawekea na kuwapa wasanii uhuru wa kubadilisha mazingira kwa manufaa ya jamii. Katika usasa, watu hujigawa kimakundi ambapo utafiti huu unayaita matabaka. Vikundi hivyo hufuata utamaduni na maadili ya jamii na hutofautiana kutoka jamii moja hadi nyingine. Hata hivyo kundi tawala wanaochukua nafasi ya tabaka la juu hunyanyasa tabaka tawaliwa yaani tabaka la chini kwa manufaa yake. Malengo ya nadharia hizi ni kuvipa vikundi vilivyotengwa nguvu kwa mfano wa hawa ni: walemavu, wanawake, watu walio na ulemavu wa ngozi na wazee ni jamii za wachache duniani. Uchunguzi huu haukujikita kwenye nadharia ya usasa kwa kigezo kwamba ukoloni mamboleo huchukua baadhi ya sifa zake na kuunda tabaka la juu, tabaka la watawala, ambao mara nyingi hunyanyasa tabaka la chini.

\section{SUALA LA UTAFITI}

\section{Suala la Utafiti}

Fasihi ni kioo cha jamii na hivyo inatarajiwa kuibua na kuelezea hali halisi katika jamii. Tafiti mbalimbali zimefanywa zinazoshughulikia vipengele vya lugha katika fasihi. Vipengele hivyo vya fasihi vinajumuisha maudhui, wahusika, fani (mitindo lugha) na mandhari. Utafiti huu ulichunguza mifumo ya jamii inayotokana na vipengele hivyo na kwa upana wake hakika imekuwa mifumo inayotokana na fasihi. Utafiti huu ulibainisha mifumo jamii ambayo inajitokeza kutokana na fasihi andishi, utanzu wa tamthilia kama malighafi ya utafiti wenyewe. Vitabu vitatu vya tamthilia vimetumika ambavyo ni: Pango (2003), Kifo Kisimani (2008) na Mstahiki Meya (2009). Baada ya kuibainisha mifumo hiyo, utafiti ulionyesha jinsi inavyoweza kuwasilisha masuala ya kijamii kupitia drama. Kadhalika, uchunguzi umeonyesha ufaulu wa waandishi wa tamthilia hizi kuyawasilisha masuala hayo katika uhalisia wake. Utafiti huu umekuwa na jukumu kuonyesha uhusiano thabiti uliopo baina ya mifumo ya jamii na vipengele vya lugha. Aidha uchunguzi huu umebainisha jinsi vipengele hivyo vimetumika kuiwasilisha mifumo ya kijamii. Mifano ya vipengele hivyo ni; maudhui, mitindo lugha, wahusika na mandhari ambavyo vimetumika kubainisha na kuwasilisha mifumo ya jamii ambalo ndilo suala letu kuu.

\section{Sababu za Uteuzi wa Mada}

Dhima kuu ya fasihi ni kuichora jamii jinsi ilivyo. Njogu na Chimerah (2006) wanasema kuwa hili linapoafikiwa jamii hupata elimu na burudani. Ili fasihi iichore jamii kikamilifu sharti itumie lugha ya kawaida waitumiayo wanajamii, iangazie uchumi na shughuli wazifanyazo ili waishi, dini zao, imani yao, itikadi wanazoziamini, matabaka yao, utawala, uongozi na siasa zao. Haya yote huwekwa mizani kwa kuipitia migogoro ya jamii, mivutano na mikinzano baina ya watu binafsi na kati ya vikundi vinavyoibuka ndani ya jamii.

Fasihi aidha hutoa mwelekeo kwa kuangazia sababu za upungufu pale unapopatikana na hupongeza pale mambo yanapofaulu. Haya ni mambo yasiyo kikomo na hupitishwa kutoka kizazi kimoja hadi kingine kimaandishi au kisimulizi. Utafiti huu umeonyesha jinsi mifumo ya kijamii inavyobainika kupitia fasihi andishi na jinsi ingetumika katika ukuaji wa jamii, mchango wake katika uimarishaji na urekebishaji wa mienendo ya wanajamii kwa kuhusishwa katika maamuzi anavyosema Wamitila (2010). Mifano ya mifumo kutoka tamthilia teule imetumika ili kuweka bayana dhana kuwa fasihi andishi husheheni mifumo jamii mbali na kuwa kielelezo kijenzi kinachochangia ukuaji na uimarishaji wa fasihi kwa ujumla. Ni kutokana na wazo hili ambapo maoni ya Hough (1969) kuwa hakuna kazi ya fasihi yoyote iliyoandikwa na kukamilika kwa wakati mmoja na kufikia kikomo yanakuwa na uzito. Hough anazidi kusema kuwa uhakiki unapofanyika kwa kazi yoyote ile, historia na mazingira ambako uhakiki ulifanyiwa ni muhimu. Vipindi vya nyakati vinapopita, wahakiki na wachunguzi wa fasihi huwa na mitazamo tofauti kwa kazi ile ile iliyoandikwa na kuhakikiwa hapo awali. Ili kazi za kisinkronia na kidaikronia ziweze kuchangia ukuaji wa fasihi, hatuna budi basi kuzitafiti kazi zenyewe upya. Uchunguzi huo mpya huziweka kando nadharia za zamani na kutumia nadharia za kisasa kama vile umitindo, usasa na usasabaadaye ili kukidhi vigezo vya fasihi kwenda na wakati ili papatikane tija na manufaa kwa jamii.

Msokile (2003) akichangia mjadala huu anatoa mifano ya mifumo ya kale iliyojengeka kutokana na nadharia za nyakati hizo. Anatoa mifano ya mashairi ya kirasmi yaliyozingatia utunzi kwa 
kufuata kanuni zilizowekwa na malenga wa kwanza kama vile vina na mizani, kinyume na mashairi ya karne za 20 na 21 yanayozingatia uhuru wa mtunzi na kutilia maanani maudhui katika utunzi kuliko maumbo. Hivyo basi, kwa maoni ya Hough na Msokile tunasema kuwa uchunguzi na uhakiki wenye tija ni ule unaochangia ukuaji wa fasihi na kutambua hatua zilizopigwa katika ukuaji huo kutoka kwa wanafasihi wengine kwa uzingatifu wa nadharia za wakati husika.

Watt (1957) anasema kwamba ili mhakiki aweze kuelezea ukweli wa jamii husika sharti aingie na kutangamana na wanajamii wengine. Anapofanya hivyo humbidi kushiriki katika mifumo jamii katika uhalisia wake na pia kubaini misingi ya kila mfumo husika. Wamitila (2010) anaongezea kuwa mifumo hiyo inapodhihirika, basi ukweli hujitokeza na hili linasaidia katika ukuaji wa jamii na fasihi kwa ujumla wake. Haya husaidia kuafiki dhima ya fasihi kuichora jamii jinsi ilivyo na uchunguzi unapochunguza mifumo hii katika tamthilia, bila shaka mhakiki anatangamana na wanajamii na anaweza kusema kuwa kwa hakika mifumo hii imo kwenye jamii. Njogu na Chimerah (2006) wakitoa maoni sawa na ya Wamitila wanasema kuwa mwanafasihi hawezi kujitenga na matukio, migogoro na mivutano iliyomo kwenye jamii yake. Fasihi kupitia kwa wahakiki inapoweka matatizo ya jamii paruwanja husaidia kuonyesha upungufu na mafanikio ya jamii na pia matarajio ya wanajamii. Upungufu huwa chanzo cha kutafuta uvumbuzi wa kuutatua kwa vizazi vilivyomo na vijavyo na mafanikio hutia wahakiki moyo na mshawasha wa kutaka kufanikiwa zaidi. Uchunguzi huu umeshughulikia mifumo katika tamthilia tatu tu. Hivyo basi utatia wanafasihi wengine mshawasha wa kufanya uchunguzi zaidi kwa kutumia tamthilia na tanzu zingine za fasihi ya Kiswahili.

Sababu nyingine ya kuichagua mada hii ni kubaini athari za mifumo jamii katika matabaka ya jamii. Kwa mujibu wa Gill (2006) matabaka hujitokeza kupitia wahusika ambao hujenga ploti na dhamira ya fasihi husika. Dhamira huletwa kutokana na maudhui. Utafiti huu umebainisha jinsi maudhui yamechangia kubadilisha mifumo duni na kuweka mifumo inayowaauni wanajamii kuinua maisha yao.

\section{NADHARIA YA UTAFITI}

\section{Misingi ya Nadharia za Utafiti}

Nadharia za uhakiki hulenga kuhakiki fasihi na kazi za kifasihi, kuzitafsiri, kuzichambua na kuziweka kwenye muktadha fulani ili kuleta uasilia wa jamii kiuchumi, kisiasa na kiusomaji (Wamitila, 2010). Uchunguzi huu umefanikishwa kwa kutumia nadharia tatu ambazo ni nadharia za Uhalisia na Umtindo kama nadharia kuu pamoja na kiunzi cha nadharia kikiwa nadharia ya Ukoloni Mamboleo.

\section{Nadharia ya Uhalisia}

Baadhi ya waasisi wa nadharia ya uhalisia ni Jule Francaic Champfleury na Stendahal wa Ufaransa, Alexander Pushkin wa Urusi na Ian Watt wa Uingereza. Uhalisia wenyewe ulijitokeza kudengua na kuupinga ulimbwende na umepitia vipindi kadhaa vilivyojitokeza kwa sura tatu nazo ni: Uhalisia hakiki, Uhalisia wa kijamaa na Uhalisiajabu. Uhalisia hakiki unahusisha fasihi ya ufaransa ikiongozwa na Stendahal karne ya kumi na nane. Jambo lililosisitizwa hapa ni waandishi kutumia fikra katika ukweli wala si ubunilizi. Njogu na Wafula (2007) wanasema kuwa uhalisia huu husawiri na kuzingatia uyakinifu katika uhakika wa maisha. Wamitila (2002) anauita uhalisia huu akisiko katika hali halisi. Anasisitiza mambo katika uhalisia hakiki kuwasilisha maisha ya jamii kwa uaminifu na usahihi mkubwa. Hii ni kuwa na maana kwamba mambo yanayoonekana kwa jamii yatolewe kwa picha halisi kwa kusawiri wahusika matukio na mandhari (Wamitila, 2006). Utafiti huu umechukua uhalisia hakiki kama msingi wake wa uchunguzi kwa sababu unahusisha utambuzi wa mifumo jamii kutoka kwa tamthilia teule lakini pia unalinganisha maisha ya wanajamii halisi kupitia wahusika waliomo kwenye tamthilia teule ambao wanawakilisha jamii zetu kwa vile fasihi ni kioo cha jamii.

\section{Mihimili ya Nadharia ya Uhalisia}

Nadharia ya uhalisia imetumika kubainisha uhalisia wa mifumo ya kijamii inavyobainika kupitia tamthilia teule. Watt (1957) anaiita nadharia hii ukweli na uyakinifu katika uzoevu wa maisha ya binadamu ilhali Ntarangwi (2004) anaiita uwakilishi halisi wa mambo katika fasihi. Mbali na 
uhalisia, uchunguzi huu umetumia pia nadharia za umtindo na ukoloni mamboleo. Baadhi ya misingi ya nadharia ya Uhalisia ni kuwa: Kihistoria ilianzia huko Uropa karne ya 19. Ntarangwi (2004) anasema kuwa jukumu kubwa la nadharia ya uhalisia ni kueleza ukweli wa maisha katika jamii na ukweli wenyewe hudhihirika kupitia shughuli za kijamii ambazo huhusisha siasa, uchumi wake, imani zao, falsafa na itikadi. Ukweli huo unaweza kuwa ukweli wa kisanaa unaohusisha mambo ya kisanaa yanayoweza kuaminika na hujumuisha maisha ya binadamu. Watetezi wa uhalisia wanasema kuwa ukweli huu katika maisha ya kawaida hudhihirika kupitia mambo yafuatayo:-

\section{Utawala na Uongozi}

Katika mfumo wa utawala na siasa za jamii, nguzo hii imepelekea kuhoji uongozi uliomo kwenye tamthilia teule. Hali ya uongozi kwenye tamthilia zaweza kupigiwa mfano kama kioo cha jamii halisi na hivyo fasihi kuwa kioo cha jamii.

\section{Masuala ya Vijana}

Kizazi na nguvu kazi ya jamii huwa ni vijana. Ili uchumi wa jamii uimarike sharti kila mwanajamii achukue nafasi yake kuujenga wakiwemo vijana. Vijana hawa wakionekana kuwa wengi kuzidi vikundi vingine vya jamii zetu katika ulimwengu wa tatu, wanakumbwa na migogoro na misukosuko mingi likiwemo suala la ndoa, dini, ajira na ukosefu wa kazi. Haya ni maoni ya Gill (2006). Utafiti huu umechunguza nafasi adimu ya vijana, huku wakiwa wamesongwa na migogoro mingi lakini ndani ya mivutano hiyo na jinsi mifumo jamii kama vile lugha wanayoitumia kwenye shughuli zao za maisha inavyobainika.

\section{Udhanifu}

Migogoro inayozuka kutokana na mtu kushindwa kuamua afuate mkondo upi wa mambo kwa mfano siasa gani, utamaduni upi, dini gani ambavyo hupelekea kuathirika kwa mifumo jamii iliyomo.

\section{Masuala ya Ukosefu wa Kazi na Ajira}

Kazi huwezesha mtu binafsi kujiimarisha kiuchumi na kupelekea jamii nzima kuinuka kimaisha. Hata hivyo pale kazini kuna unyanyasaji, mishahara midogo na mibovu na mazingira mabovu ya kufanyia kazi. Kupitia tamthilia teule utafiti umeyavalia njuga masuala haya na kuona mifumo jamii iliyojitokeza.

\section{Masuala ya Jinsia}

Utafiti umechunguza haki za makundi yaliyopuuzwa katika jamii kama vile makundi ya walemavu, vijana na wanawake. Mihimili yote ya nadharia ya uhalisia kwa njia moja au nyingine imetoa mchango kwa lengo la kuafikia madhumuni ya utafiti huu. Kwa mifano: mhimili wa utawala na uongozi ulichunguza utawala na uongozi uliomo kwenye tamthilia teule. Kutokana na uchunguzi huo, utafiti ulijenga maudhui ya uongozi mbaya ambao pia ulichangia kudhihirisha mfumo wa utawala na siasa. Mihimili ya masuala ya vijana na yale ya ukosefu wa ajira na kazi imechangia kuiweka mifumo ya uchumi wa jamii, elimu na utabaka wazi. Migogoro baina ya kizazi cha vijana na kile cha kale hutokana na kile kinachoaminiwa na kila kkundi na hili lilibainisha uwepo wa mifumo ya imani na hata dini na ule wa utamaduni kueleweka.

\section{Nadharia ya Umtindo}

Umtindo kwa mujibu wa Katie Wales (1990) katika a dictionary of Stylistics ni tawi la isimu linaloshughulikia uchanganuzi wa maneno katika usemi ili kuleta maana. Anazidi kusema kuwa mbali na maendelezo na mipangilio ya maneno, umtindo huangazia pia tafsiri ya usemi ili kukamilisha mawasiliano yenye maana. Njogu na Chimerah (2011) wanaona umtindo kama mojawepo ya nadharia za kijamii. Nadharia nyingine ya kijamii ni umithilishaji iliyotumiwa na Plato kwa kigezo kuwa sanaa ni umithilishaji wa hali fulani Ulimwenguni. Wanazidi kusema kuwa sanaa humithilishwa na kile kinachoonekana na msanii na wala sio kile cha asilia yaani dhana ya daima dawamu. Njogu na Chimerah wanaiona nadharia ya umtindo kuwa inayozingatia mtindo wa kazi ya sanaa bila kushughulika na maudhui .Umitindo hujikita katika uchambuzi wa kitarakimu, miundo ya maneno, sarufi na maana zake. Uchunguzi huu hutokana na imani kuwa kitovu cha kazi yoyote ile ni mtindo sababu mtindondio unaosheheni maudhui. Jukumu la mhakiki anayetumia nadharia hii ni uchambuzi wa kimaelezo na kitarakimu kuhusu msamiati, 
sentensi, kuhesabu takriri za vokali na konsonanti, mizani na mambo mengine ya kisarufi. Uhakiki wa kazi kimtindo hufichua ujumi wa kazi ya sanaa kutokana na vitendo vya wahusika, aina za wahusika na mandhari ya uhusika.

\section{Mihimili ya Nadharia ya Umtindo}

Mihimili ya nadharia ya Umtindo kwa mujibu wa Michael Burke (2014) ni pamoja na:-

i. Picha inayohusu wahusika katika uhusika wao na mandhari wanayobadilishabadilisha ili kufikia kifani au upeo wa hadithi ijenge taswira kupitia mazungumzo na mdahalo baina ya hao wahusika, fikira zao, matumizi ya mitindo lugha kati yao; kwa mfano kinaya, matumizi ya ishara na mitindo yote iweze kuwasilisha maudhui na dhamira iliyokusudiwa na mwandishi.

ii. Mfuatano wa mawazo kutoka sentensi moja hadi nyingine uhusishe uradidi wa konsonanti, miundo ya maneno katika sentensi ilete maana, urudiaji wa vokali fulanifulani, taswira na sarufi katika kiimbo ziweze kuleta matamshi yanayojenga sarufi.

iii. Fasihi iwe na jukumu la kuiathiri jamii kutokana na tungo zitokanazo na utamaduni wa jamii husika (Njogu na Chimerah, 1999)

iv. Fasihi na historia zifungamane kulingana na mazingira ya jamii, kuwa na maana kwamba historia ya jamii itokane na mivutano na migogoro iliyomo kwenye jamii kupitia wahusika.

v. Wahusika wa fasihi fulani wawakilishe matabaka yote ya jamii na matabaka hayo yaweze kuingiliana.

vi. Matumizi lugha yawe ya umbuji wa kuhakiki jinsi mwandishi ameteua lugha kuwasilisha ujumbe wake na kuleta kifani naye msomaji kutoa kauli za jumla zinazounganisha mada na kipengele cha usimulizi yaani mhusika, kitendo na maudhui. Kauli hizo zifunganishwe na kutolewa mifano ili kuhukumu kazi ya fasihi.

Mihimili yote ya umtindo imechangia katika kupata matokeo ya utafiti. Uchunguzi wa mtindo katika, wahusika na madhari uliwezesha kujenga maudhui ili kubaini mifumo jamii iliyotokana nayo. Tamathali za usemi zilisaidia kuwaelewa wahusika katika mazingira yao na hili linadokeza uwepo wa mifumo ya utabaka, utawala na siasa na uchumi wa jamii. Mifano ya mandhari yaliyojitokeza ni yale ya mjini, mashambani na pangoni. Kauli za kiutawala zilizotolewa kwenye mandhari hayo ziliathiri jamii katika tamthilia za uchunguzi na kuwezesha ubainifu wa mifumo jamii.

Nadharia ya umtindo imekuwa na nafasi kubwa na kuwa msingi wa kuchambua vipengele vya lugha yaani mtindo uliotumiwa na waandishi wa tamthilia teule nas baadaye kuweza kudondoa mifumo jamii kutokana na matumizi lugha, wahusika na mandhari.

\section{Nadharia ya Ukoloni Mamboleo}

Nadharia hii imetumika kuonyesha uongozi mbaya kupitia viongozi wahusika. Pia nadharia hii imetumika kuonyesha vile mfumo wa matabaka hujitokeza na mifumo lugha inayotofautiana baina ya matabaka.

\section{Mihimili ya Nadharia ya Ukoloni Mamboleo}

Kwa mujibu wa Nkrumar (1965), vigezo vinavyojenga mihimili ya nadharia ya Ukoloni Mamboleo ni pamoja na:

i. Ushirika kati ya wakoloni na nchi walizozitawala kwa manufaa ya kiuchumi. Masharti waliyoweka wakoloni yaendelee kuheshimiwa na nchi zinazojidai kuwa huru ili wapate faida nyingi za malighafi ya viwanda vyao na manufaa mengine kama vile soko kwa bidhaa walizounda katika viwanda hivyo.

ii. Mataifa yaliyopewa uhuru yakubali kutawaliwa na taasisi za kimataifa kama vile makampuni makubwa na benki ambazo huwawekea wenyeji masharti magumu lakini yasiyo budi kuheshimiwa ili kuwafaidi wakoloni. Taasisi hizi huitwa za kimataifa.

iii. Nchi zilizopewa uhuru zitumie jeshi lao kulinda maslahi ya wakoloni dhidi ya wananchi wake ili wasije wakaathiri maslahi ya wakoloni. Nchi hizo zilizotawaliwa kuwa 
na vituo vya kijeshi kutoka jeshi la wakoloni. Lengo lao ni kuthibiti na kulinda maslahi yao.

iv. Wakoloni husaidia serikali zinazotii masharti yao kuingia mamlakani ambazo huwa vivuli kuwashughulikia wao badala ya kushughulikia maslahi ya wananchi wake.

\section{MBINU ZA UTAFITI}

Muundo wa utafiti huu ulikuwa wa kiuchanganuzi. Baada ya kuvisoma vitabu vingine mbalimbali vya fasihi vilivyosaidia kuelewa kile waandishi wengine wameandika kuihusu mada. Mtafiti aliweka data kwenye rekodi za uchunzaji zilizotumika kudondoa maudhui na mtindo jinsi ulivyotumika katika tamthilia teule. Dhamira ya kufanya hayo ilikuwa kufanikisha ubainifu wa mifumo jamii. Mifumo ya kijamii ikibainika, madhumuni ya utafiti yalikuwa yameafikiwa. Hizi zote zimekuwa mbinu za kimakusudi na mbali na kuibainisha mifumo ya kijamii, utafiti umeangalia vile mifumo hiyo inaathiri maisha ya kila siku katika jamii. Uathiri wa maisha ya jamii zilizomo kwenye tamthilia ni mfano tu wa yale yanayofanyika kwenye jamii zetu na hivyo kimekuwa kiwakilishi tosha cha fasihi kuwa kioo cha jamii.

Utafiti huu ulitumia asilimia kumi ya tamthilia zote zilizokadiliwa kuaandikwa nchini Kenya na Tanzania. Utafiti huu umetumia tamthilia 26 kwa sababu hizi ndizo zimeandikwa Afrika Mashariki na Kati kufikia kipindi kinachozungumziwa yaani, 2000 - 2014. Asilimia 10 ya hizo inatupa kifani cha tamthilia mbili nukta sita (2.6). Kwa mujibu wa Mugenda na Mugenda (1999), asilimia kumi ya kundi lote ni kiwakilishi tosha cha utabia, sifa na uwakilishi wa jumla wa kundi kubwa linalotafitiwa. Aidha, uchunguzi huu umejikita Kenya na Tanzania kwa sababu kuwa hapa ndipo sera na sheria za nchi hizi zimekipa Kiswahili kipao mbele. Katika Utabia ni kuwa zote ni tamthilia ambapo Kifo Kisimani sawa na Pango zina maonyesho kumi kila moja na ile ya Mstahiki Meya ina maonyesho kumi na mawili. Tamthilia hizi zimewekwa pamoja kwa kufanya uteuzi wa kimakusudi wa kuibainisha mifumo jamii inavyodhihirika katika fasihi andishi kwa kutumia utanzu wa tamthilia.

\section{Uwasilishaji wa Matokeo ya Uchunguzi}

Ripoti hii ya uchunguzi imewasilishwa kwa njia ya kimaelezo. Maelezo yenyewe yanahusu mada na yana vigezo, viwango na sifa za ukubalifu kuwa ndiyo matokeo ya uchunguzi. Matokeo hayo yamejadiliwa kwa kina na kwa hakika yameangazia madhumuni ya utafiti. Aidha, matokeo hayo yameonyesha wazi kuwa nadharia zilizoteuliwa katika uchunguzi huu ziliafiki vigezo vyote kusaidia kuyaafikia madhumuni. Nadharia ya umtindo ilikuwa msingi wa kutambua mitindo lugha, wahusika katika uhusika wao na mandhari ambayo yalichangia kutambua mifumo jamii. Matukio katika maigizo yalitumia hali halisi kuwasilisha ujumbe wa yale yanayofanyika kwenye jamii zetu.

\section{MATOKEO YA UTAFITI}

Katika utafiti huu, ni bayana kwamba mifumo ya kijamii ambayo imejitokeza kupitia kwa fasihi andishi imebainishwa. Tafiti za awali zilizojadiliwa zimeendeshwa kwa lengo la kubaini maudhui yanayojitokeza na matumizi na mitindo lugha katika fasihi andishi na nafasi ya wahusika katika mandhari mbalimbali. Utafiti huu umeenda hatua mbele na kuibainisha mifumo jamii inayojitokeza kutokana na vipengele hivi vya lugha. Utafiti ulijadili baadhi ya maoni ya wanafasihi kuhusu majukumu ya fasihi yakiwemo yale ya kuelimisha na kutumbuiza, maoni yao kuhusu mifumo ya kijamii na misingi ambayo mifumo hiyo hujengeka, na jinsi utafiti huu ungebainisha mifumo hiyo. Kadhalika utafiti umeangazia umuhimu wa utafiti huu na upeo na mipaka ya utafiti wenyewe.

Baadhi ya sababu za kufanya utafiti huu zimejadiliwa kwa kina. Hata hivyo mbali na jukumu la kiusomi, utafiti huu una nafasi kuzindua vyombo vya dola na utawala kuwafanya viongozi walio mamlakani kuwatumikia wananchi waliowachagua kwa uaminifu wakiwa na lengo la kuinua maisha ya jamii kwa ujumla. Hili likiwezekana, basi pengo linaloendelea kuwa kubwa baina ya matabaka ya jamii litapungua hasa baina ya maskini na matajiri. Pengo hilo likipungua, basi mivutano ya jamii inayoshuhudiwa huenda ikapungue pia. 
Katika kufanikisha utafiti, vyenzo za kufanikisha uchunguzi huu ambazo ni vipengele vya lugha zimejadiliwa. Vipengele hivyo vimejadiliwa kwa kina vikiwemo vile vya maudhui, mtindo kama vile mitindo lugha, wahusika na mandhari. Mihimili ya nadharia za uchunguzi huu zimejadiliwa kwa kina. Nadharia hizi ni nadharia ya uhalisia, nadharia ya umtindo na nadharia ya ukoloni mamboleo. Ubora wa nadharia hizi kuafiki malengo ya utafiti umefanyiwa hukumu na kupatikana kuwa hakika zimesaidia kutimiza madhumuni ya utafiti.

Ukusanyaji na uchanganuzi wa data umejadiliwa na hatimaye njia za uwasilishaji matokeo. Sura hii pia imeangazia jinsi ya kulinda haki za kila mmoja aliyehusika katika utafiti kwa njia moja ama nyingine katika. Hizi zote ni mbinu za kimakusudi za kukamilisha utafiti. Ufasiri na mjadala kuhusu maudhui na mtindo kama vyenzo za kutambua mifumo jamii vimejadiliwa. Mifano ya maudhui kama vile uongozi mbaya, unyanyasaji. ufisadi nk. yamejadiliwa kwa kina na hasa kuonyesha jinsi maudhui hayo yalitumika katika utambuzi wa mifumo jamii kutoka tamthilia teule.

Mbali na maudhui, sehemu hii imejadili mitindo kama vile mitindo lugha ikiwemo methali, tashbihi, kinaya, misemo, jazanda nk. na kuonyesha vile inajenga hisia zinazochangia katika utambuzi wa mifumo jamii. Aidha, wahusika na uhusika kwenye mandhari vimejadiliwa kama sehemu ya mtindo na kuoyesha vile vinachangia katika kutimiza madhumuni ya utafiti. Hatimaye katika sehemu hii mifumo jamii imejadiliwa na kuonyesha nafasi ya nadharia teule kufanikisha uchunguzi huu.

Mfumo wa utawala na siasa umeonyesha siasa zilizomo kwenye tamthilia baina ya viongozi na wanajamii. Tunapata viongozi hawa wakipingwa na wananchi walio wengi kwa sababu ya uongozi mbaya. Imebainika kuwa ukweli uliojitokeza kwenye tamthilia haupo mbali na viongozi wetu wa kisiasa na hivyo kuonyesha jinsi waandishi wa tamthilia teule walivyofaulu kuandika fasihi iliyomo kwenye jamii zetu. Katika mfumo wa uchumi, utafiti umeonyesha kuwa mara nyingi wale viongozi wa kisiasa ndio pia husimamia vyanzo vya kiuchumi katika jamii zetu. Utafiti unahimiza usimamizi huu uwe wa wazi na ukweli ili kuepusha ufisadi na migogoro ambayo haingeshuhudiwa. Haya yatawezekana ikiwa wananchi watahusishwa katika shughuli za kila siku za uchumaji mali kupitia ajira na pia uwazi katika ughafi wa raslimali.

Tamaduni ni muhimu kwa jamii yoyote ile. Hata hivyo kupitia mfumo wa utamaduni tumejifunza kuwa tamaduni hasi zilizopitwa na wakati kama vile tohara kwa wasichana na kuwatenga wanawake katika nyadhifa za uongozi zapashwa kutupiliwa mbali. Tamaduni chanya ambazo zinasaidia ukuaji wa jamii zipongezwe na kuimarishwa. Katika mfumo wa imani na dini, utafiti unahimiza viongozi wa kidini kuwa wa kweli na wawe mstari wa mbele kutetea ukweli na haki katika jamii na sio wale wanaolenga kujinufaisha binafsi kutoka kwa wanajamii. Hatimaye katika mfumo wa elimu, utafiti unapendekeza elimu kwa wote bila ubaguzi na kuwe na usawa katika viwango vyote vya elimu ya jamii. Katika kila hatua ya utafiti huu, mihimili ya nadharia zilizotumika kufanikisha utafiti imeangaziwa na jinsi inavyoingiliana na matukio ya uchunguzi ili kutoa mchango na kufanikisha utafiti wenyewe. Hatimaye uchunguzi huu umetoa mapendekezo ya tafiti zaidi kuhusu mifumo jamii.

\section{HITIMISHO NA MAPENDEKEZO}

Matumaini yangu ni kuwa fasihi ikiwa kioo cha jamii, utafiti huu utakuwa wa manufaa kwa wasomi wote, serikali zetu zikiwemo zile za kaunti na serikali kuu na wanajamii kwa ujumla ili kutimiza dhima ya fasihi ya kuelimisha jamii. Azma kuu ya utafiti huu ilikuwa kubainisha mifumo jamii kupitia fasihi andishi na kudhihirisha kuwa si yote yaliyoandikwa yanakuwa bunilizi bali mengine huwa ya uhalisia. Fasihi ikiwa kioo cha jamii yapashwa kuwa na uwezo wa kuchangia maendeleo ya jamii na kuweza kuinua viwango vya maisha ya wanajamii.

Kutokana na matokeo ya uchunguzi huu, utafiti umebainisha vile mifumo jamii inavyodhihirika kupitia fasihi andishi, utanzu wa tamthilia. Matumaini ya mtafiti ni kuwa utafiti zaidi wapashwa kufanywa kuihusu mifumo hii. Utafiti unapendekeza uchunguzi kufanywa kuhusu manufaa ya mifumo jamii kutokana na fasihi andishi na pia kuibaini mifumo jamii katika riwaya. 


\section{MAREJELEO}

Arege, T. (2011). Mstahiki Meya, chapa ya kwanza. Nairobi. Vide-Muwa Publishers.

Burke, M (2014). The Routledge Handbook of Stylistics. Newyork: Routledge.

Gill, R. (2006). Mastering English Literature (3 ${ }^{\text {rd }}$ Edition). Newyork: Macmillan

Hough, G. (1969). Style and stylistics (First edition). London: Routeledge.

Mberia, K. (2008). Kifo Kisimani. (Chapa ya kumi na sita). Nairobi: Marimba Publications

Mohamed, S. A. M. (2001). Babu Alipofufuka. Nairobi: J.K.F.

Msokile, M. (2003). Fasihi ya Kiswahili (Chapa ya kwanza). Nairobi: E.A.E.P.

Mugenda, M. (1999). Research on Target Population, First edition. Nairobi: Kenyatta University Press.

National Council of Law (2010). The Kenyan Constitution. Nairobi. Government Printers Ltd.

Njogu, K. \& Chimerah, R. (1999). Ufundishaji wa Fasihi; Nadharia na Mbinu. Nairobi: Jomo Kenyatta Foundation.

Njogu, K. \& Chimerah, R. (2006). Ufundishaji wa Fasihi (Chapa ya pili). Nairobi: J.K.F.

Njogu, K. \& Chimerah, R. (2011). Ufundishaji wa Fasihi (Chapa ya tatu). Nairobi: Jomo Kenyatta Foundation.

Njogu, K. \& Wafula, R. (2007). Nadharia za Uhakiki wa Fasihi (Chapa ya kwanza). Nairobi: J.K.F.

Nkrumah, K. (1965). Neo-colonialism; The Last Stages of Imperialism. London: Thomas Nelson and Sons Ltd.

Ntarangwi, M. (2004). Uhakiki wa kazi ya Fasihi ( $1^{\text {st }}$ Edition). Rock Island, IL: Augustina College.
Obeng-Odoom, F. (2013). Africa's failed economic development trajectory: a critique. African Review of Economics and Finance, 4(2), 151175.

Wafula, R. M. (2003). Uhakiki wa Tamthilia na Maendeleo yake. Nairobi: J.K.F

Wales, K. (1990). A dictionary of Stylistis. New York: Routledge.

Wamitila, K. W. (2001), Kamusi ya Methali za Kiswahili (Toleo la kwanza). Nairobi: Longhorn Publishers.

Wamitila, K. W. (2002). Uhakiki wa Fasihi, Misingi na Vipengele vyake (Toleo la kwanza). Nairobi: Longhorn Publishers.

Wamitila, K. W. (2003), Pango (Toleo la kwanza). Nairobi: Focus publishers.

Wamitila, K. W. (2003). Kamusi ya Fasihi; Istilahi na Nadharia (Toleo la kwanza). Nairobi: Focus Publishers.

Wamitila, K. W. (2004). Kichocheo cha Fasihi (Toleo la pili). Nairobi: Phoenix Publishers,

Wamitila, K. W. (2006). Uhakiki wa Fasihi (Misingi na Vipengele vyake). Nairobi: Phoenix publishers.

Wamitila, K. W. (2010). Kanzi ya Fasihi; Misingi ya Uchanganuzi (Chapa ya pili). Nairobi: VideMuwa Publications.

Watt, I. (1957). Rise of the Novel ( $1^{\text {st }}$ Edition). New York: Penguin. 